\title{
Wasf3 Deficiency Reveals Involvement in Metastasis in a Mouse Model of Breast Cancer
}

\author{
Haiyan Qin, ${ }^{*}$ Sumin Lu, ${ }^{*}$ Muthusamy Thangaraju, ${ }^{\dagger}$ and John K. Cowell ${ }^{*}$
}

From the Georgia Cancer Center, $*$ and the Department of Biochemistry and Molecular Biology, ${ }^{\dagger}$ Augusta University, Augusta, Georgia

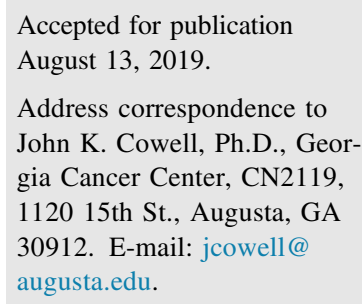

\begin{abstract}
The WASF3 gene has been implicated in cancer cell movement, invasion, and metastasis by regulating genetic pathways important in these processes. Invasion and metastasis assays, however, are largely centered on xenograft models in immune-compromised mice. To facilitate analysis of the role of WASF3 in the spontaneous development of cancer cell metastasis, we generated a Wasf3 null strain by deleting exons 4 and 5, which encode essential motifs for Wasf3 function. On exposure to cre-recombinase a stop codon is generated immediately downstream in exon 6. Using a cytomegalovirus (CMV)-cre strain, Wasf3 constitutively was inactivated, which led to viable mice with no visible morphologic or behavioral abnormalities. There was no abnormal development or function of the mouse mammary gland in the Wasf3 null mice and brain development was normal. In the mouse mammary tumor virus (MMTV)-driven polyoma middle-T oncogene strain, which shows early onset breast cancer development and metastasis, Wiskott-Aldrich syndrome protein family member 3 (Wasf3) is up-regulated in metastatic lesions. When this oncogene was introduced onto the Wasf3-null background, although metastasis was observed in these mice, there was a reduction in the number and size of metastatic lesions in the lungs. These data provide evidence for a role in WASF3 in the development of metastasis in a spontaneous model of breast cancer. (Am J Pathol 2019, 189: 2450-2458; https://doi.org/10.1016/j.ajpath.2019.08.012)
\end{abstract}

The Wiskott-Aldrich syndrome protein family member 3 (WASF3/WAVE3) $^{1}$ is part of a three-member gene family that orchestrates actin polymerization through specific domains that bind monomeric actin and actin related protein (ARP)2/3 proteins. $^{2,3}$ WASF proteins normally are autoinhibited through a protein complex that binds to the WASF regulatory region. ${ }^{4-6}$ In response to extracellular stimuli, the regulatory complex is released and verprolin/cofilin/ acidic domains in the WASF proteins are exposed, allowing recruitment of actin monomers for polymerization. ${ }^{7,8}$ Each of the WASF proteins has been implicated in various aspects of cell movement involving reorganization of membrane structures such as ruffles, invadopodia, and lamellipodia. 9,10 The same actin polymerization process is involved in cancer cell invasion and metastasis, but only WASF3 has been shown to have a central role in this process because inactivation of WASF3 in highly metastatic breast cancer cells leads to suppression of invasion and metastasis but inactivation of WASF1 and WASF2 does not. ${ }^{11}$ The in vitro invasion phenotype has been correlated with metastasis in vivo and loss of WASF3 function in human cancer cells suppresses metastasis in vivo. ${ }^{12-14}$ Overexpression of WASF3 in nonmetastatic cells leads to increased invasion and metastasis in in vivo experimental metastasis models. ${ }^{15}$ These in vitro and mouse xenografts studies, therefore, have clearly implicated WASF3 in the promotion of metastasis and targeting its function suppresses metastasis in xenograft models. ${ }^{14}$ Gene expression studies in human cancers have further implicated WASF3 in metastasis development with positive correlations between expression levels and unfavorable outcomes in breast cancer patients. ${ }^{16}$ The majority of functional analyses, however, have been performed in mouse model systems, where cells are manipulated in vitro and then metastasis is evaluated in xenograft studies in vivo in immunocompromised mice.

To investigate the effects of deleting WASF3 in spontaneous models of breast cancer metastasis, a

Supported by the Georgia Cancer Center (J.K.C.).

Disclosures: None declared. 
conditional Wasf3 null mouse strain was generated and it was shown that homozygous inactivation of Wasf3 leads to viable mice with no observable morphologic or behavioral abnormalities. To investigate the role of Wasf 3 in spontaneous metastasis, an oncogene was introduced into the Wasf3 null mouse. The majority of analyses of Wasf3 function in metastasis have centered on breast cancer; therefore, a mouse model of spontaneous metastasis was considered as follows: i) in which the incidence of the primary tumor was completely penetrant, ii) that showed a small latency period, iii) in which metastases developed in at least $90 \%$ of animals, iv) in which metastases developed with a short latency period, and v) in which all mice carried the transgene. The mouse mammary tumor virus-driven polyoma middle-T oncogene (PyMT) model ${ }^{17}$ develops palpable tumors by approximately 5 weeks of age in all animals and shows metastasis by approximately 4 to 6 months of age. ${ }^{17}$ Although human breast cancers do not typically express PyMT, this oncoprotein is responsible for dysregulation of many of the genes that are hallmarks of breast cancer including $M Y C C, S R C, E R B B 2$, integrins, cyclin D, $R A S$, and $P I 3 K$, as well as loss of estrogen receptor and progesterone receptor during progression. ${ }^{18}$ This wholesale dysregulation of key breast cancer-associated genes likely is responsible for the early onset and complete penetrance of breast cancer in this model. The tumors that develop in these mice also go through the same histologically defined changes seen in human tumors from benign hyperplasia to carcinoma. ${ }^{17,18}$ Here, the PyMT oncogene was introduced onto a Wasf3 null background and a significant reduction in metastatic lung lesions, without elimination of metastasis, was shown in the Wasf 3 null mice.

\section{Materials and Methods}

\section{Generation of the Wasf3 Conditional Knockout Mouse Strain}

The Wasf 3 conditional knockout vector was generated using homologous recombination strategies. ${ }^{19}$ Homology arms flippase recognition target were isolated from the RP23$134 \mathrm{~N} 18$ bacterial artificial chromosome (BAC) genomic clone using Red recombination as described previously. ${ }^{20}$ After homologous recombination, the vector contained exons 4,5 , and 6 of the Wasf 3 gene with approximately $5 \mathrm{~kb}$ of flanking sequence on either side. Two loxP sites and a chloramphenicol resistance gene $(\mathrm{Cat})$ were inserted into the vector using PCR. Chloramphenicol-resistant clones were isolated carrying the pStart-WASF3-loxp/loxP vector. Flippase recognition target (FRT) and the neomycin resistance gene (neo), together with its phosophoglycerate kinase (PGK) promoter, were inserted into the vector as described previously. ${ }^{20} \mathrm{~A}$ herpes simplex virus thymidine kinase (HSV-tk) cassette also was inserted as a negative selection marker using Gateway recombination. ${ }^{20}$ The sequence- verified targeting vector then was electroporated into C57BL/6 embryonic stem (ES) cells (at the Emory University Core facility) and clones subsequently were selected using G418 and fialuridine (FIAU) and sequence-verified for the homologous recombination event. ES cells were microinjected into C57BL/6-derived blastocysts to generate the targeted mouse. Subsequently, the blastocysts were surgically transferred to C57BL/6 pseudopregnant foster mothers to allow the embryos to come to term. Chimeric males with the high ES cell contribution were crossed with C57BL/6 females. Deletion of Wasf3 was achieved by crossing to CMV-cre-expressing mice.

The conditional Wasf $3^{\text {flox/flox }}$ mice are available from the Jackson Laboratory (stock 033353; Bar Harbor, ME).

\section{Molecular Studies}

DNA preparation and PCR amplification were performed using standard procedures.

Western blot was performed as described previously. ${ }^{10}$ Cells were lysed in ice-cold RIPA buffer $[25 \mathrm{~mol} / \mathrm{L}$ Tris$\mathrm{HCl}, \mathrm{pH} 7.5,150 \mathrm{~m} \mathrm{NaCl}, 1 \mathrm{~m}$ EDTA, $1 \%$ Triton X-100 (Fischer, Fair Lawn, NJ), and a mixture of proteases inhibitors] and analyzed by SDS-PAGE, followed by immunoblotting. Briefly, after blocking with $5 \%$ milk, the membranes were probed with primary antibodies at $4^{\circ} \mathrm{C}$ overnight. The membrane was washed and then incubated with secondary antibodies at room temperature for 1 hour. Immunoreactive proteins were developed with enhanced chemiluminescence reagents (Pierce, Rockford, IL). The following antibodies were used: mouse monoclonal anti- $\beta$ actin antibody (A5316; Sigma, St. Louis, MO) and WASF3 antibody (2806S; Cell Signaling, Danvers, MA). Horseradish-peroxidase-conjugated anti-mouse and anti-rabbit were used as secondary antibodies. Signal was detected and developed using the SuperSignal West Pico Chemiluminescent Substrate system (Thermo Scientific, Waltham, MA).

\section{Histopathology}

\section{Whole Mount Analysis}

The fourth inguinal glands were dissected at the indicated development times and spread on a glass slide. After fixation for 2 to 4 hours in Carnoy's fixative, the tissues were hydrated and stained in carmine alum overnight. ${ }^{21}$ Slides then were dehydrated, cleared in xylene, and mounted.

\section{Tissue Sections}

Tissues were fixed in $4 \%$ neutral formalin, dehydrated in alcohol, and embedded in paraffin using standard procedures. Six-micrometer sections then were prepared, dewaxed, stained with hematoxylin and eosin, and mounted on microscope slides. 


\section{Statistical Analysis}

Individual data points were plotted using Prism8 GraphPad software (Graphpad Software Inc., San Diego, CA) in which the means and SDs were calculated. Significance was determined using a two-tailed $t$-test using Excel version 16.25 (Microsoft, Redmond, WA).

\section{Results}

\section{Generation of a Wasf3 Conditional Knockout Vector}

The Wasf 3 start codon is located in exon 3 and, by deleting exons 4 and 5, a stop codon is generated at the beginning of exon 6 (Figure 1A), leading either to loss of the resultant mRNA through nonsense-mediated decay pathways or a severely truncated (52 amino acid) protein lacking both the important WASF regulatory domain and the verprolin/ cofilin/acidic domains essential for WASF3 function in actin polymerization. The targeting construct (Figure 1B) was generated in the pSTART-K vector ${ }^{19}$ following a previously described approach. ${ }^{20}$

The targeting vector was analyzed extensively using PCR and restriction enzyme digestion where the expected sizespecific fragments were confirmed. Finally, the various essential regions of the vectors were sequenced individually to confirm the correct vectors had been produced. The sequence-verified targeting vectors then were electroporated into C57BL/6 ES cells and clones were selected using G418 and FIAU. These cells were microinjected into B6-derived blastocysts, which were transferred surgically to B6 pseudopregnant foster mothers to allow the embryos to come to term. Chimeric progeny were identified by PCR genotyping and males estimated to contain $>80 \%$ ES cells were used to generate the F1 generation.

\section{Generation of Wasf3 Null Mice}

Wasf3 heterozygous flox/+ mice were bred to generate homozygous conditional (flox/flox) null mice, which were verified through PCR analysis using tail-derived DNA. These mice then were bred to a strain expressing cre recombinase driven by the CMV promoter, ${ }^{22}$ which provides global knockout of the Wasf 3 gene. Genotyping of offspring (Figure 1, C and D) showed that the ratio of null and heterozygous null mice followed a Mendelian distribution and that the Wasf3 null mice survived to term. Over the first 2 months of observation there was no obvious change in structural or behavioral phenotypes and the mice were the same size as normal mice. Because Wasf 3 is expressed strongly in neurons in the brain, ${ }^{23}$ attention was provided to their general psychomotor activity and the ability to feed, grip, and walk, for which there was no difference compared with either heterozygous littermates or wild-type mice. High-resolution histopathologic analysis of the cortex of the null mice also was performed, which showed no abnormalities in cortical structure (Supplemental Figure S1).

Because Wasf3 is expressed highly in the brain, Western blot was used to analyze the presence of Wasf3 in the isolated cortex from the parental and knockout mice. Western blot analysis of the knockout mice showed an absence of the protein, confirming that targeted deletion of exons 4 and 5 led to loss of Wasf3 function as predicted (Figure 1E).

\section{Wasf3 Loss Does Not Affect Mammary Gland Development}

Because the aim of this project was to evaluate the role of Wasf3 in spontaneous development of metastases in the mouse mammary tumor virus-PyMT mouse model of breast cancer, we first examined whether loss of Wasf3 function had any effect of normal mammary gland development. It was clear from the breeding studies, however, that the Wasf3 null female mice gave birth to normal litter sizes ( 8 to 10 pups) and nursed the pups in the same way normal mice would. The fact that the pups survived and grew to adulthood indicated that the mammary glands were functioning normally. Histopathologic analysis of the gland in normal virgin mice is shown in Figure 2, showing normal development within the gland generating a normal ductal tree with multiple tertiary branches. Pregnant females showed normal secondary and tertiary ductal development with alveologenesis and branching of the ducts and lactating animals showed typical ductal expansion (Figure 2). Thus, there was no evidence of any abnormal development or function of the mammary gland as a result of loss of Wasf3.

\section{Loss of Wasf3 Reduces Metastatic Lesions in the Lung}

The mouse mammary tumor virus-PyMT mice develop tumors spontaneously in all animals after 3 to 4 months and metastasis occurs in the vast majority of these animals within 6 to 8 months. This metastatic ability of the primary tumor cells in the mammary gland was shown by analysis of vimentin and E-cadherin, two markers of the epithelium-tomesenchyme transition. Mammary gland tumors showed down-regulation of E-cadherin and increased levels of vimentin, consistent with the epithelium-to-mesenchyme transition phenotype (Supplemental Figure S2).

To determine whether loss of Wasf 3 affected spontaneous metastasis of breast cancer tumor cells, the mouse mammary tumor virus-PyMT oncogene was introduced onto the Wasf3 null background, using the breeding protocol described in Materials and Methods. Mice were genotyped for PyMT+WASF3-, and parallel cohorts with parental PyMT mice were maintained to compare the effect of loss of Wasf3 function on metastasis. The number and size of tumors in PyMT and PyMT/Wasf $3^{-1-}$ mice was studied. The lungs from the same two cohorts of mice were removed at sacrifice to evaluate metastatic load. Macroscopic analysis of the unfixed lungs from the PyMT mice frequently 
A

Wasf3-WT $\rightarrow 501$ aa

\begin{tabular}{|c|c|c|c|c|c|c|c|}
\hline & $\begin{array}{l}\text { Intron } \\
16403 \text { bp }\end{array}$ & $\begin{array}{l}\text { Intron } \\
1310 \mathrm{bp}\end{array}$ & $\begin{array}{l}\text { Intron } \\
2074 \text { bp }\end{array}$ & $\begin{array}{l}\text { Intron } \\
5303 \text { bp }\end{array}$ & $\begin{array}{l}\text { Intron } \\
5657 \text { bp }\end{array}$ & $\begin{array}{l}\text { Intron } \\
1150 \mathrm{bp}\end{array}$ & $\begin{array}{l}\text { Intron } \\
1640 \text { bp }\end{array}$ \\
\hline E3 & E4 & E5 & E6 & E7 & E8 & E9 & E10 \\
\hline ATG......A & GT......G & TC.......AG & A......AAG & GAA.......AG & G.......GG & G......GG & GA......TGA \\
\hline $133 b p$ & 135 bp & $154 \mathrm{bp}$ & 118 bp & $176 \mathrm{bp}$ & $267 \mathrm{bp}$ & 365 bp & 158 bp \\
\hline
\end{tabular}

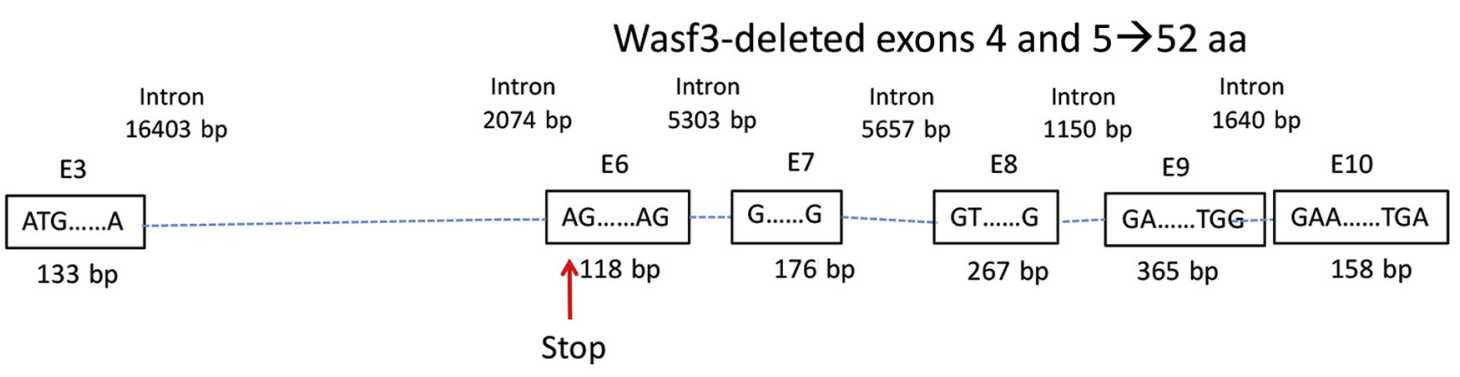

B

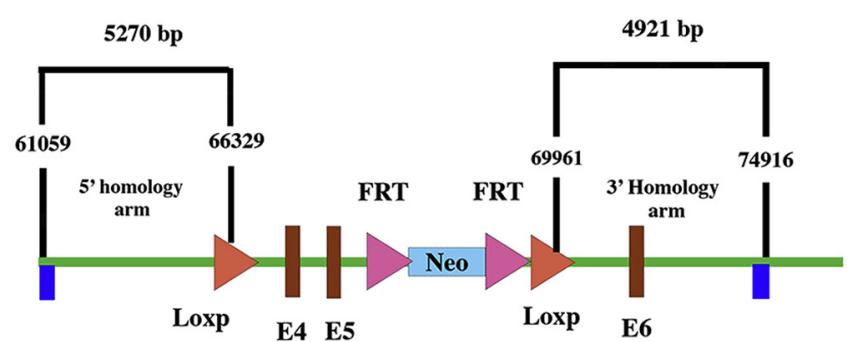

D

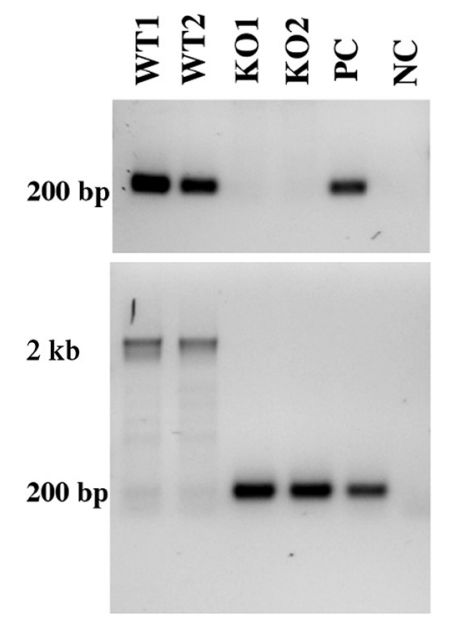

C
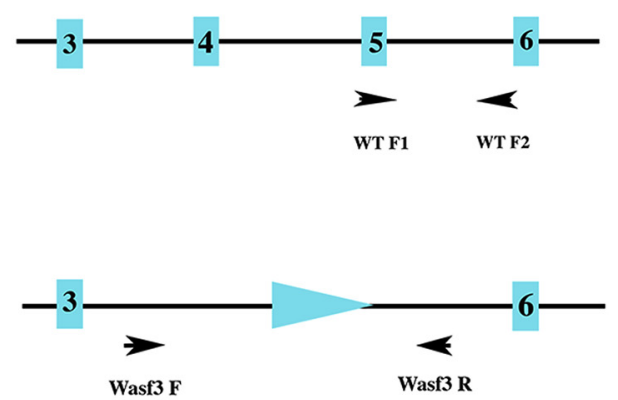

$\mathbf{E}$

\section{Fetal brain}
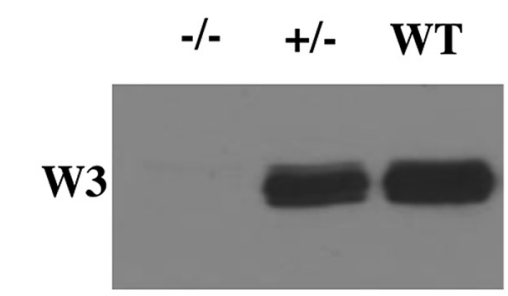

Lgi1

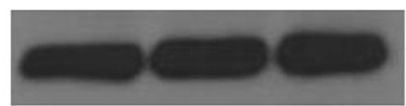

Figure 1 Generation of the Wasf3 null mouse strain. A: Schematic of the exon structure of the Wasf3 gene showing the consequences of deleting exons 4 and 5, leading to a stop codon in exon 6. B: The targeting construct to generate the deletion through homologous recombination. C: Genotyping for the wildtype (WT) allele involves using primers (WTF1) located in exon 5 and WTR2 located downstream in intron 5 (WT forward: $5^{\prime}$-TCTAAAGGCCTTGGACCTTGACACTGGCTGGA-3'; WT reverse: $5^{\prime}$-TGACTTTGACAGCAAGCCGATCGA-3'), which produces a 226-bp fragment only in the WT mice. When exons 4 and 5 are deleted, no PCR product is generated with these primers. Using primers WASF3F and WASF3R, designed from within introns 3 and 5 (forward: $5^{\prime}-A G C A G T A A-$ CATCTTGACTGACAGCTCAGGCA-3'; reverse: 5'-TGACACATGCAACCAGCATAGCAA-3'), a 266-bp fragment is generated across the deletion site. D: Analysis of mice after expression of Cre recombinase using the primers described in $\mathbf{C}$ shows the WT PCR product in wild-type mice but not the PCR fragment across the deletion site. Instead, a 2214-bp fragment is generated including exons 4 and 5. Using the WASF3F and WASF3R PCR primers, the 266-bp fragment is present only in the null mice. E: Western blot analysis of proteins from the brains of Wasf3 null mice (-/-) shows the absence of the Wasf3-specific product compared with that seen in heterozygous floxed mice (+/-) or WT mice. The Lgi1 protein, which is highly expressed in the mouse cortex, was used as the loading control. E, exon; F, forward; FRT, flippase recognition target; KO, knockout; NC, negative control with no template; PC, positive control from the original founder mice; R, reverse. 

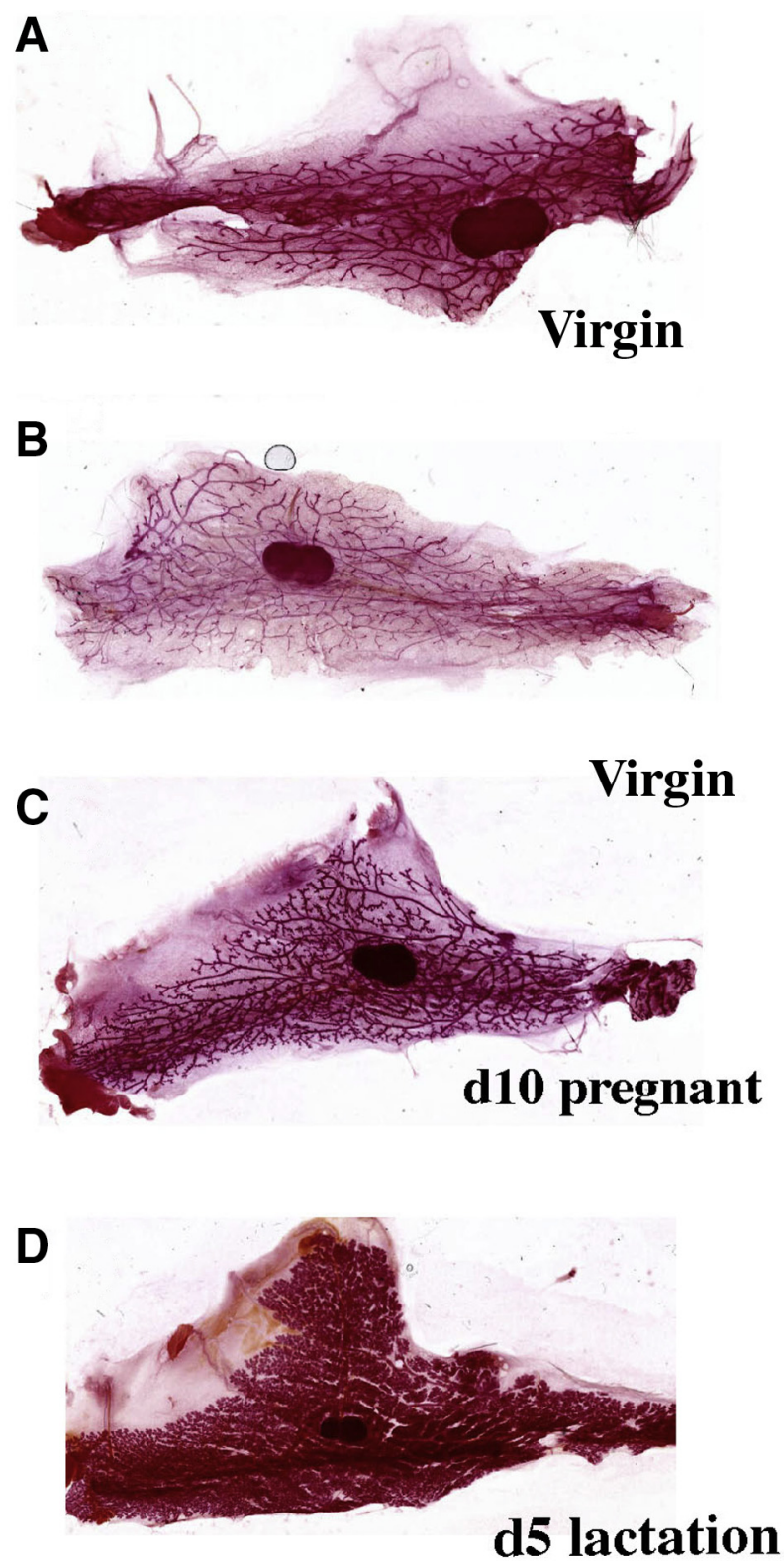

Figure 2 Development of the mammary gland in Wasf3 null mice. A and B: Whole mount, carmine alum-stained mammary glands from Wasf3 null virgin mice show the typical ductal structure. $\mathbf{C}$ and $\mathbf{D}$ : Ten-day-old pregnant females show ductal expansion (C), which is even more accentuated in day 5 lactating females (D). d, day.

showed the presence of large surface nodules (Supplemental Figure S3). None of the lungs from the PyMT-W3 ${ }^{-1-}$ mice showed these macroscopic lesions, suggesting, at least at this level of resolution, that the loss of Wasf 3 could suppress metastatic dissemination. The lungs then were fixed in formalin and subjected to histologic analysis. For each lung, two sections were prepared, with the first cut through the center of the lung sample and the second section was prepared $100 \mu \mathrm{m}$ deeper into the lung. Each section was stained with hematoxylin and eosin and the presence and size of tumors were evaluated.
The variation in tumor incidence seen on the lung surface (Supplemental Figure S3) was reflected in the histologic analysis, which showed large tumors (Figure 3A) distributed throughout the lungs in $40 \%$ of the PyMT mice. In other animals, the lungs showed variation in both the size and number of tumors (Figure 3A), although all mice showed at least one metastatic lesion, in one case, only a single small lesion was detected. Histology of the lungs from the PyMT/ $\mathrm{Wasf}^{-1-}$ mice, however, showed no metastatic lesions in the lungs in 5 of $13(38 \%)$ mice. In the other mice, there were generally fewer, smaller tumors in the lungs (Figure 3B). Because the extent of metastasis likely is dependent on the tumor load at the primary site in the breast, it was shown that although there was some heterogeneity in the latency with which the primary tumors arose in the mammary fat pads, typically the parental PyMT mice developed large tumors within 120 to 140 days in the majority of mammary fat pads. The same was true for the PyMT/Wasf $3^{-/}$mice and there was no significant difference in the number of primary tumors in the two cohorts (Figure 4A). When the number of individual tumor foci was counted in the sections from the two different depths in the lung, there was no significant difference within each genotype (Figure 3B). When the total number of tumors identified in the tissue sections was compared between genotypes, there was a significant reduction $(P=0.0255)$ in the number of metastatic lesions in the PyMT/Wasf $3^{-/-}$mice (Figure 4B). Next, the area of each lung occupied by tumor metastases was measured. ImageJ software version 1.48 (NIH, Bethesda, MD; http://imagej.nih.gov/ij) was used to measure the total area in each tissue section and was expressed as a percentage of the total area of the lung present in the section. In the PyMT mice there was clearly a bimodal distribution in the extent of the tumor area in the lungs, with several mice showing $>90 \%$ of the lungs occupied by metastases, which skewed the data. When these mice were excluded from the analysis, there was a marginally significant $(P=0.057)$ reduction in the overall area of the lungs occupied by tumor in the PyMT/ Wasf $^{-1-}$ mice (Figure 4C).

Analysis of WASF3 in primary human breast cancers has suggested an association with more aggressive tumors such as the claudin-low subgroup, which has the poorest survival. ${ }^{16}$ To determine whether Wasf 3 is up-regulated in the metastatic lesions in the mouse models described here, Wasf3 expression levels were analyzed in primary breast tumors from the PyMT and the PyMT-Wasf $3^{-1-}$ mice and their metastases. None of the primary breast tumors that developed in either genotype $(n=5)$ showed detectable Wasf3 expression (Figure 4D). Analysis of Wasf3 expression levels in the metastatic lung lesions in the PyMT mice $(n=3)$, compared with the primary breast tumors, showed up-regulation of WASF3 (Figure 4D), supporting the suggestion seen in human studies that increased WASF3 expression correlates with metastatic progression. As expected, neither the primary tumors nor the metastatic lesions 

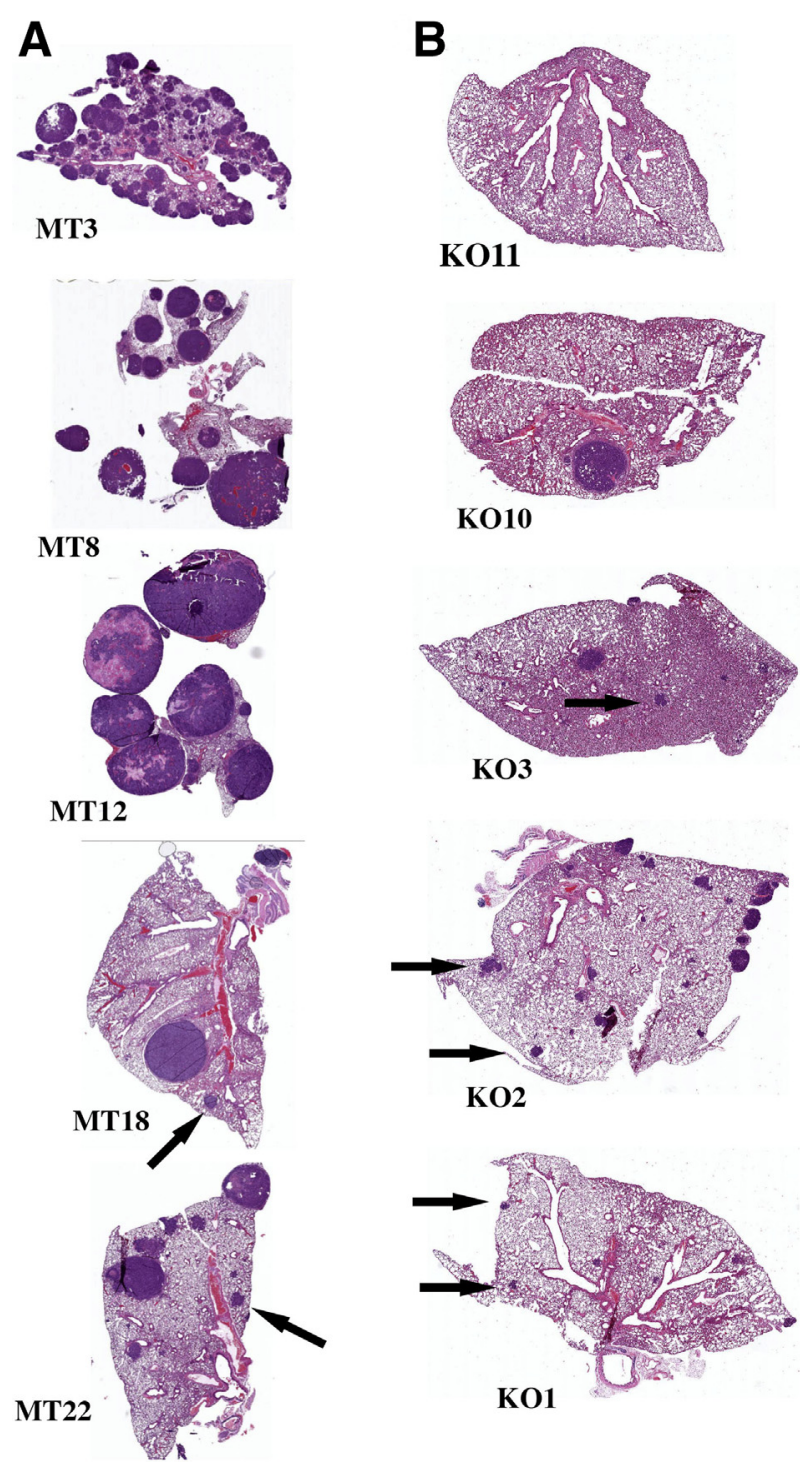

KO2

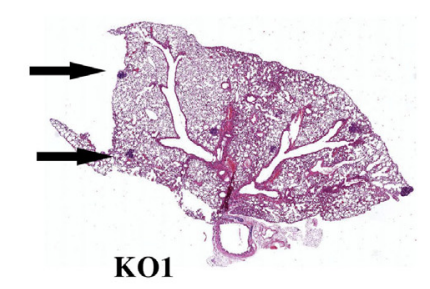

Figure 3 Histologic analysis of lungs from PyMT and PyMT/Wasf3 null mice. A: Hematoxylin and eosin-stained sections from representative PyMT mice show the variation in tumor number and size of metastatic lesions in the lungs. The tumors in these mice are typically large (MT3, MT8, MT12), although smaller tumors also are present (arrows). B: Analysis of lungs from PyMT/Wasf3 null mice typically show only fewer, small tumors (arrows), with the exception of the single large tumor seen in mouse K010 and the absence of tumors in mouse K011.

in the lungs of the PyMT/Wasf $3^{-/-}$mice expressed Wasf3 (Figure 4E).

\section{Discussion}

Evidence suggesting a role of WASF3 in the development of metastasis has been established through the following: i) correlative studies in human cancers ${ }^{16}{ }^{1}$ ii) in vitro manipulation of WASF3 expression levels, ${ }^{10,13,24}$ and iii) pharmacologic studies targeting WASF3 function in vivo. ${ }^{14}$ The in vitro studies provide a more robust model to evaluate the molecular mechanisms behind WASF3 regulation of invasion and motility, which is supported consistently in xenograft studies using the genetically manipulated cells. Together, these studies have shown that WASF3 function has a significant role in suppressing metastasis. These studies, however, consistently showed that loss of WASF3 function does not lead to total elimination of invasion and metastasis, possibly because shRNA knockdown used in these studies could not totally eliminate expression. The absence of WASF3 expression in mammary glands, although not affecting normal breast development or restructuring ductal expansion during pregnancy, leads to a significant suppression of the number and size of the metastases, although cannot totally prevent metastatic dissemination to the lungs. The mechanism of metastasis typically begins with intravasation into blood vessels and/or the lymphatic system. A zebrafish model of human breast cancer cells showed that metastasis ${ }^{25}$ is suppressed as a result of WASF3 knockdown. This is likely owing to the regulation of metastasis-promoting genes such as various matrix metallopeptidases (MMPs), which are regulated by WASF $3 .^{26,27}$ This may provide one explanation for the reduced number of metastases, possibly through delays in the release of metastasizing cells as a result of their reduced ability to intravasate, and why the tumors in the lungs are generally smaller or absent. Successful development of metastatic lesion in sites such as the lungs also depends on extravasation from the blood vessels into the distant organ, which is facilitated by the same mechanisms leading to intravasation. In experimental models of metastasis in which the cells are injected directly into the blood vessels and therefore bypass intravasation, there has been a reduction in the number and size of metastatic lesions in animals receiving cells in which WASF3 has been suppressed, ${ }^{12}$ again possibly explaining why primary breast cancer cells in our PyMT model show smaller and fewer metastatic lesions in the lungs. Once established in the lungs, however, these metastatic lesions can expand because loss of WASF3 does not have a significant effect on cell proliferation. ${ }^{28,29}$ Therefore, the number of metastatic lesions, rather than their size, is the best indication of metastatic potential. It is also possible that, even though WASF3 exerts a significant effect on the metastasis process, other genetic changes in cancer cells can compensate for this loss and use other pathways to promote metastasis. The apparently random nature of the acquisition of these compensatory events also could contribute to the heterogeneity in latency and hence size of the metastatic lesions.

Although the involvement of WASF3 in the suppression of breast cancer metastasis has been established using model cell systems, it is clear from the studies presented here that loss of WASF3 does not prevent metastasis in the PyMT model of breast cancer. There is a major difference, however, in investigating the role of WASF3 in highly evolved cell lines compared with primary breast epithelial cells. The up-regulation and activation of WASF3 

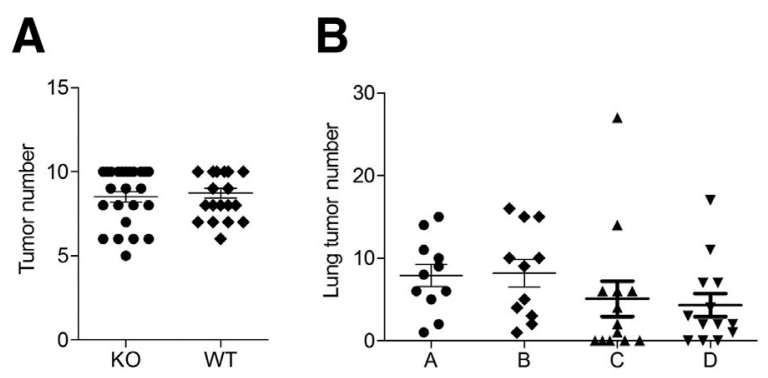

C

D
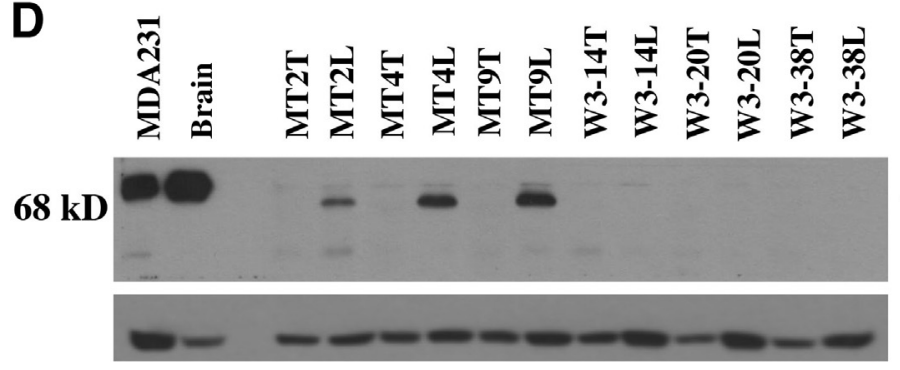

WASF3

$\mathbf{E}$

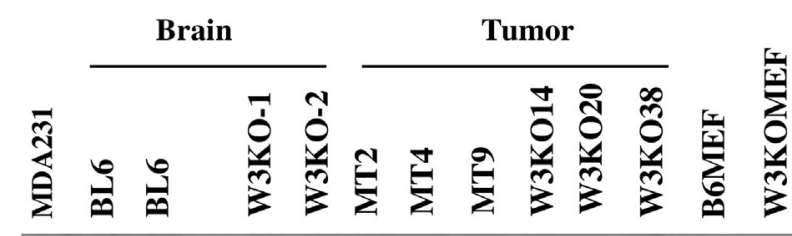

$68 \mathrm{kD}$

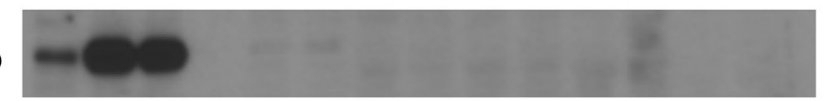

WASF3

ACTB

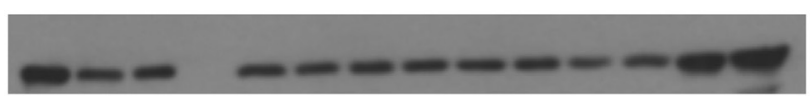

\section{ACTB}

promotes metastasis and this can be achieved through activation of receptors such as human epidermal growth factor receptor $2,^{30}$ interleukin 6 signaling through the Janus kinase/signal transducers and activators of transcription pathway, ${ }^{31}$ and other growth factors (such as $\left.P D G F R^{26}\right)$. As a result, one of the major consequential effects was on gene expression levels, particularly the down-regulation of KISS1, which is involved in NF- $\mathrm{BB}$ signaling through $Z E B 1 .^{15,27}$ It therefore seems that deregulation of a number of pathways is required for WASF3 to function as a metastasis suppressor and, although beyond the scope of this study, whether the same conducive genetic environment is present in the PyMT tumors, remains to be determined. Clearly, from the study of primary human breast cancers, however, up-regulation of WASF3 is a late event in the transformation process, ${ }^{16}$ which is also the case in the PyMT model.

Although the majority of early studies focused on breast cancer, in our case, because of the availability of convenient in vivo models to evaluate molecular mechanisms, ${ }^{12}$ there is evidence that WASF3 also can influence metastasis and outcome in other cancer types. We have shown, for example, that suppression of WASF3 in prostate cancer cells also leads to reduced invasion and metastasis $^{13}$ as well as in colon cancer cells ${ }^{29}$ and melanoma (unpublished). Beyond these studies, there are reports comparing WASF3 expression levels and metastasis/ outcome in, for example, hepatocellular carcinoma ${ }^{32}$ and small cell lung cancer, ${ }^{33}$ but in these largely correlative studies it was not possible to determine the specific role of WASF3. Functional relationships between WASF3 and metastasis, however, have been established in ovarian cancer ${ }^{34}$ gastric cancer, ${ }^{35}$ pancreatic cancer, ${ }^{36}$ and osteosarcoma. ${ }^{37}$ The availability of the Wasf $3^{\text {flox/flox }}$ strain, therefore, provides the opportunity to investigate the role of WASF3 in other cancer models such as the transgenic adenocarcinoma of the mouse prostate (TRAMP) model for prostate cancer $^{38}$ or the multiple intestine neoplasia (MIN) mouse for colon cancer. ${ }^{39}$

The WASF family of genes comprises three members that share homologous regulatory and verprolin/cofilin/acidic domains related to actin polymerization dynamics. Knockdown of WASF1 and WASF2 in highly metastatic breast cancer cells does not affect invasion, whereas knockdown of WASF3 has a profound influence on invasion and metastasis. ${ }^{11}$ It appears, therefore, that the other members of this 
gene family determine overlapping, but nonredundant, functions, which is reflected in the phenotypes of mice null for these genes. Wasf3 null mice appear to have normal development. In contrast, the WASF1 null mice survive to term but show neurologic defects that affect brain development and die in the first month of life. ${ }^{40,41}$ Wasf 2 null mice are embryonic lethal, showing distinct phenotypes associated with abnormal brain development. ${ }^{42}$ Brain development in Wasf3 null mice, however, is apparently normal, suggesting it has a more restricted role in development.

\section{Supplemental Data}

Supplemental material for this article can be found at http://doi.org/10.1016/j.ajpath.2019.08.012.

\section{References}

1. Sossey-Alaoui K, Su G, Malaj E, Roe B, Cowell JK: WAVE3, an actin-polymerization gene, is truncated and inactivated as a result of a constitutional $\mathrm{t}(1,13)(\mathrm{q} 21, \mathrm{q} 12)$ chromosome translocation in a patient with ganglioneuroblastoma. Oncogene 2002, 21:5967-5974

2. Takenawa T, Suetsugu S: The WASP-WAVE protein network: connecting the membrane to the cytoskeleton. Nat Rev Mol Cell Biol 2007, 8:37-48

3. Derivery E, Gautreau A: Generation of branched actin networks: assembly and regulation of the N-WASP and WAVE molecular machines. Bioessays 2010, 32:119-123

4. Gautreau A, Ho HY, Li J, Steen H, Gygi SP, Kirschner MW: Purification and architecture of the ubiquitous wave complex. Proc Natl Acad Sci U S A 2004, 101:4379-4383

5. Stovold CF, Millard TH, Machesky LM: Inclusion of Scar/WAVE3 in a similar complex to Scar/WAVE1 and 2. BMC Cell Biol 2005, 6:11

6. Chen Z, Borek D, Padrick SB, Gomez TS, Metlagel Z, Ismail AM, Umetami J, Billadeau DD, Otwinowski Z, Rosen MK: Structure and control of the actin regulatory WAVE complex. Nature 2010, 468: $533-538$

7. Pollard TD, Borisy GG: Cellular motility driven by assembly and disassembly of actin filaments. Cell 2003, 112:453-465

8. Insall RH, Machesky LM: Actin dynamics at the leading edge: from simple machinery to complex networks. Dev Cell 2009, 17:310-322

9. Takenawa T, Miki H: WASP and WAVE family proteins: key molecules for rapid rearrangement of cortical actin filaments and cell movement. J Cell Sci 2001, 114:1801-1809

10. Sossey-Alaoui K, Li X, Ranalli TA, Cowell JK: WAVE-3 mediated cell migration and lamellipodia formation are regulated downstream of P13-kinase. J Biol Chem 2005, 280:21748-21755

11. Teng Y, Bahassan A, Dong D, Hanold LE, Ren X, Kennedy EJ, Cowell JK: Targeting the WASF3-CYFIP1 complex using stapled peptides suppresses cancer cell invasion. Cancer Res 2016, 76: 965-973

12. Sossey-Alaoui K, Safina A, Li X, Vaughan MM, Hicks DG, Bakin AV, Cowell JK: Down-regulation of WAVE3, a metastasis promoter gene, inhibits invasion and metastasis of breast cancer cells. Am J Pathol 2007, 170:2112-2121

13. Teng Y, Ren M, Cheney R, Sharma S, Cowell JK: Inactivation of the WASF3 gene in prostate cancer cells leads to suppression of tumorigenicity and metastases. Br J Cancer 2010, 103:1066-1075

14. Cowell JK, Teng Y, Bendzunas NG, Ara R, Arbab AS, Kennedy EJ: Suppression of breast cancer metastasis using stapled peptides targeting the WASF regulatory complex. Cancer Growth Metastasis 2017, 10:1-9
15. Teng Y, Mei Y, Hawthorn LA, Cowell JK: WASF3 regulates miR200 inactivation by ZEB1 through suppression of KISS1 leading to increased invasiveness in breast cancer cells. Oncogene 2013, 33: 203-211

16. Prat A, Parker JS, Karginova O, Fan C, Livasy C, Herschkowitz JI, He X, Perou CM: Phenotypic and molecular characterization of the claudin-low intrinsic subtype of breast cancer. Breast Cancer Res 2010, 12:R68

17. Guy CT, Cardiff RD, Muller WJ: Induction of mammary tumors by expression of polyomavirus middle $\mathrm{T}$ oncogene: a transgenic mouse model for metastatic disease. Mol Cell Biol 1992, 12:954-961

18. Lin EY, Jones JG, Li P, Zhu L, Whitney KD, Muller WJ, Pollard JW: Progression to malignancy in the polyoma middle $\mathrm{T}$ oncoprotein mouse breast cancer model provides a reliable model for human diseases. Am J Pathol 2003, 163:2113-2126

19. Wu S, Ying G, Wu Q, Capecchi M: A protocol for constructing gene targeting vectors: generating knockout mice for the cadherin family and beyond. Nat Protoc 2008, 3:1056-1076

20. Silva J, Qin H, Cowell JK: Selective inactivation of LGI1 in neuronal precursor cells leads to cortical dysplasia in mice. Genesis 2019, 57 : e23268

21. Robinson GW, McKnight RA, Smith GH, Hennighausen L: Mammary epithelial cells undergo secretory differentiation in cycling virgins but require pregnancy for the establishment of terminal differentiation. Development 1995, 121:2079-2090

22. Koni PA, Joshi SK, Temann U, Olson D, Burkly L, Flavell RA: Conditional vascular cell adhesion molecule 1 deletion in mice. J Exp Med 2001, 193:741-754

23. Suetsugu S, Miki H, Takenawa T: Identification of two human WAVE/SCAR homologues as general actin regulatory molecules which associate with the Arp2/3 complex. Biochem Biophys Res Commun 1999, 260:296-302

24. Sossey-Alaoui K, Li X, Cowell JK: c-ABL-mediated phosphorylation of WAVE3 is required for lamellipodia formation and cell migration. J Biol Chem 2007, 82:26257-26265

25. Teng Y, Xie X, Walker S, Saxena M, Kozlowski D, Mumm J, Cowell JK: Evaluating human cancer cell metastasis in zebrafish. BMC Cancer 2013, 13:453

26. Sossey-Alaoui K, Ranalli TA, Li X, Cowell JK: WAVE3 promotes cell motility and invasion through the regulation of MMP-1, MMP-3 and MMP-9- expression. Exp Cell Res 2005, 308:135-145

27. Teng Y, Liu M, Cowell JK: WASF3 promotes MMP9 expression through down-regulation of the KISS1 metastasis suppressor gene in breast cancer cells. Int J Cancer 2011, 129:2825-2835

28. Teng Y, Qin H, Bahassan A, Bendzunas NG, Kennedy EJ, Cowell JK: The WASF3-NCKAP1-CYFIP1 complex is essential for breast cancer metastasis. Cancer Res 2016b, 76:5133-5142

29. Teng Y, Ngoka L, Cowell JK: Promotion of invasion by mutant RAS is dependent on activation of the WASF3 metastasis promoter gene. Genes Chromosomes Cancer 2017, 56:493-500

30. Teng Y, Pi W, Wang Y, Cowell JK: WASF3 provides the conduit to facilitate invasion and metastasis in breast cancer cells through HER2/HER3 signaling. Oncogene 2016, 35:4633-4640

31. Teng Y, Ghoshal P, Ngoka L, Mei Y, Cowell JK: Critical role of the WASF3 gene in JAK2/STAT3 regulation of cancer cell invasion. Carcinogenesis 2013, 4:1994-1999

32. Ji Y, Li B, Zhu Z, Guo X, He W, Fan Z, Zhang W: Overexpression of WAVE3 promotes tumor invasiveness and confers an unfavorable prognosis in human hepatocellular carcinoma. Biomed Pharmacother 2015, 69:409-415

33. Wu J, Wang GC, Chen XJ, Xue ZR: Expression of WASF3 in patients with non-small cell lung cancer: correlation with clinicopathological features and prognosis. Oncol Lett 2014, 8:1169-1174

34. Lu J, Wang SL, Wang YC, Wu YN, Yu X, Zhao WZ, Wang JH: High WAVE3 expression correlates with proliferation, migration and invasion in human ovarian cancer. Oncotarget 2017, 8: 41189-41201 
35. Yue Z, Feng W, Xiangke L, Liuxing W, Qingxia F, Jianbo G: WAVE3 promotes epithelial-mesenchymal transition of gastric cancer through upregulation of snail. Cancer Gene Ther 2014, 21: 499-506

36. Huang S, Huang C, Chen W, Liu Y, Yin X, Lai J, Liang L, Wang Q, Wang A, Zheng C: WAVE3 promotes proliferation, migration and invasion via the AKT pathway in pancreatic cancer. Int J Oncol 2018, $53: 672-684$

37. Shen L, Wang P, Yang J, Li X: MicroRNA-217 regulates WASF3 expression and suppresses tumor growth and metastasis in osteosarcoma. PLoS One 2014, 9:e109138

38. Greenberg NM, DeMayo F, Finegold MJ, Medina D, Tilley WD, Aspinall JO, Cunha GR, Donjacour AA, Matusik RJ, Rosen JM: Prostate cancer in a transgenic mouse. Proc Natl Acad Sci U S A 1995, 92:3439-3443
39. Moser AR, Pitot HC, Dove WF: A dominant mutation that predisposes to multiple intestinal neoplasia in the mouse. Science 1990, $247: 322-324$

40. Soderling SH, Langeberg LK, Soderling JA, Davee SM, Simerly R, Raber J, Scott JD: Loss of WAVE-1 causes sensorimotor retardation and reduced learning and memory in mice. Proc Natl Acad Sci U S A 2003, 100:1723-1728

41. Dahl JP, Wang-Dunlop J, Gonzales C, Goad ME, Mark RJ, Kwak SP: Characterization of the WAVE1 knock-out mouse: implications for CNS development. J Neurosci 2003, 23:3343-3352

42. Yan C, Martinez-Quiles N, Eden S, Shibata T, Takeshima F, Shinkura R, Fujiwara Y, Bronson R, Snapper SB, Kirschner MW, Geha R, Rosen FS, Alt FW: WAVE2 deficiency reveals distinct roles in embryogenesis and Rac-mediated actin-based motility. EMBO J 2003, 22:3602-3612 